\title{
Hes1 and Hes5 Activities Are Required for the Normal Development of the Hair Cells in the Mammalian Inner Ear
}

\author{
Azel Zine, ${ }^{1}$ Alexandre Aubert, ${ }^{1}$ Jiping Qiu, ${ }^{1}$ Stavros Therianos, ${ }^{1}$ Francois Guillemot, ${ }^{2}$ Ryoichiro Kageyama, ${ }^{3}$ \\ and Francois de Ribaupierre ${ }^{1}$ \\ 1/nstitute of Physiology, University of Lausanne, 1005 Lausanne, Switzerland, 2Institut de Genetique et de Biologie \\ Moleculaire et Cellulaire, 67404 Illkirch, Centre de l'Université de Strasbourg, France, and ${ }^{3}$ Institute for Virus Research, \\ Kyoto University, Kyoto 606-8507, Japan
}

\begin{abstract}
The mammalian inner ear contains two sensory organs, the cochlea and vestibule. Their sensory neuroepithelia are characterized by a mosaic of hair cells and supporting cells. Cochlear hair cells differentiate in four rows: a single row of inner hair cells (IHCs) and three rows of outer hair cells (OHCs). Recent studies have shown that Math1, a mammalian homolog of Drosophila atonal is a positive regulator of hair cell differentiation. The basic helix-loop-helix (bHLH) genes Hes 1 and Hes5 (mammalian hairy and Enhancer-of-split homologs) can influence cell fate determination by acting as negative regulators to inhibit the action of bHLH-positive regulators. We show by using reverse transcription-PCR analysis that Hes1, Hes5, and Math1 are expressed in the developing mouse cochleae. In situ hybridization revealed a widespread expression of Hes1 in the greater epithelial ridge (GER) and in lesser epithelial ridge (LER)
\end{abstract}

regions. Hes5 is predominantly expressed in the LER, in supporting cells, and in a narrow band of cells within the GER.

Examination of cochleae from Hes $1^{-1-}$ mice showed a significant increase in the number of IHCs, whereas cochleae from Hes5 ${ }^{-1-}$ mice showed a significant increase in the number of OHCs. In the vestibular system, targeted deletion of Hes 1 and to a lesser extent Hes5 lead to formation of supernumerary hair cells in the saccule and utricle.

The supernumerary hair cells in the mutant mice showed an upregulation of Math1. These data indicate that Hes1 and Hes5 participate together for the control of inner ear hair cell production, likely through the negative regulation of Math1.

Key words: Hes1; Hes5; Math1; basic helix-loop-helix (bHLH) transcriptions factors; mouse mutant; cochlea; utricle; saccule; hair cell differentiation
Regulatory cascades of positive and negative basic helix-loophelix (bHLH) transcription factors play essential roles in the generation of numerous types of neurons from a homogenous population of ectodermal progenitor cells in the mammalian nervous system (Kageyama and Nakanishi, 1997; Lee, 1997). The inner ear initially forms as a thickening of the ectoderm, termed the otic placode in the hindbrain. The otic placode gives rise to neurons of the VIIIth cranial nerve and invaginates to become the otocyst, from which the organ of Corti and vestibular organs will develop (Van de Water, 1983; Torres and Giraldez, 1998). The sensory epithelia of these organs consist of mechanoreceptive hair cells, supporting cells, and nerve endings. In the mouse cochlea, terminal mitoses occur between embryonic day 13 (E13) and E14 (Ruben, 1967), and differentiating hair cells can first be identified on E15 in the base (Anniko, 1983; Lim and Anniko, 1985). Histological studies suggest that, during cochlea morphogenesis, inner hair cells (IHCs) derive from progenitor cells located in the greater epithelial ridge (GER) that is medial to the future organ of Corti, whereas outer hair cells (OHCs) derive from the distal progenitor cells in the lesser epithelial ridge

Received Nov. 27, 2000; revised April 2, 2001; accepted April 4, 2001.

This research was supported by Swiss National Science Foundation Grant 3156897.99. We thank Drs. Mark Mooseker for his gift of anti-myosinVIIa, Jane Johnson for anti-Math1 antibody, Thomas Van de Water for comments and suggestions, and Luc Pellerin for laboratory facilities.

Correspondence should be addressed to Dr. Azel Zine, Institut de Physiologie, 7 Rue du Bugnon, CH-1005 Lausanne, Switzerland. E-mail: azel.zine@iphysiol. unil.ch.

S. Therianos's present address: Center on Aging and Developmental Biology, University of Rochester Medical Center, Rochester, NY 14642.

Copyright $\odot 2001$ Society for Neuroscience $0270-6474 / 01 / 214712-09 \$ 15.00 / 0$
(LER) (Lim and Rueda, 1992). The time course of hair cell development includes cell fate commitment-determination, initial differentiation, maturation, and acquisition of stereociliary bundles (Kelley et al., 1993; Fekete, 1996; Torres and Giraldez, 1998). Recently, two evidences regarding initial determinationdifferentiation of mammals cochlear hair cells have been established. First, the involvement of the Notch1 signaling pathway in regulating the number of progenitor cells that develop as hair cells, contributing to the generation of the regular cochlear mosaic (Lanford et al., 1999; Zine et al., 2000a). Second, the requirement of the bHLH transcription factor Math1 as a positive regulator of the specification of hair cells in the mouse inner ear (Bermingham et al., 1999; Zheng and Gao, 2000).

The negative bHLH transcription factors Hes1 and Hes5 homologs to the products of Drosophila hairy and Enhancer-of-split [E(spl)] (Akazawa et al., 1992; Sasai et al., 1992) have been demonstrated to affect cell fate determination by inhibiting the action of bHLH-positive regulators during the development of the CNS (Kageyama and Nakanishi, 1997).

In addition, previous studies reported that both Hes 1 and Hes5 expression is induced by Notch activation (Jarriault et al., 1995, 1998; Ohtsuka et al., 1999). Although a recent study demonstrated that Hes1 acts as a negative regulator of IHC differentiation (Zheng et al., 2000), none have been shown to be required for the control of $\mathrm{OHC}$ differentiation. The accurate control of both IHCs and OHCs is necessary for the generation of the regular mosaic of hair cells and supporting cells within the mammalian cochlea. Here we examined by, using reverse transcription (RT)-PCR analysis, the expression of Hes1, Hes5, and Math1 
genes in the developing mouse cochlea. Using in situ hybridization, Hes 1 and Hes 5 mRNA expression patterns were examined in E18 and newborn cochleae.

The roles of Hes 1 and Hes 5 in hair cell differentiation were investigated by analyzing the developing inner ear from mice that lack both Hes1 and Hes5 genes.

\section{MATERIALS AND METHODS}

Animals. Compound Hes 1 and Hes5 embryos and early neonatal [postnatal day 0 (P0)] mutants were obtained from intercrosses of Hes1 ${ }^{+/-}$; Hes $5^{+/-}$or Hes $1^{+/-}$;Hes $5^{-1-}$ mice. These mutant mouse lines have been described previously (Ishibashi et al., 1995; Ohtsuka et al., 1999; Cau et al., 2000). E0.5 was defined as noon of the day that vaginal plug was observed. Inner ear tissues were collected from E16-E18 embryos or immediately after birth, because Hes $1^{-1-}$ mice die during gestation or within $1 \mathrm{~d}$ after birth (Ishibashi et al., 1995). Genotyping of the embryos and P0 mice was performed by PCR with tail DNA.

$R T-P C R$. Sensory epithelia were dissected out from 10-14 cochleae of five developmental stages from E12.5 to newborn (P0). RT-PCR was performed and controlled as described previously (Zine et al., 2000b) with few modifications. Total RNA was isolated using TRIZOL (Life Technologies, Gaithersburg, MD). Polyadenylated RNA was purified from total RNA through oligo-dT cellulose separation (Amersham Pharmacia Biotech, Uppsala, Sweden). mRNA was reverse transcribed using Superscript II RNase free reverse transcriptase (Life Technologies).

For PCR amplification, the typical thermocycle profile was as follows: $5 \mathrm{~min}$ at $95^{\circ} \mathrm{C}, 45 \mathrm{sec}$ at $58^{\circ} \mathrm{C}$, and $1.5 \mathrm{~min}$ at $72^{\circ} \mathrm{C}$ for 40 cycles. The PCR products were separated on a $1 \%$ agarose gel, stained with ethidium bromide, purified, and sequenced. The primers for RT-PCR analysis are as follows: for Hes1, upstream CAGCCAGTGTCAACACGACAC and downstream TCGTTCATGCACTCGCTGAG; for Hes5, upstream CGCATCAA CAGCAGCATAGAG and downstream TGGAAGTGGT AAA GCAGCTTC; for Math1, upstream AGTGACGGAGAGTTTTCCCC and downstream CTGCAGCCGTCCGAAGTCAA; and for glyceraldehyde-3-phosphate dehydrogenase (GAPDH), upstream GTCATCATCTCCGCCCCTTCTGC and downstream GATGCCTGCTTCACCACCTTCTTG.

In situ hybridization. Cochleae were dissected from E18 and P0 inner ears and fixed in $4 \%$ paraformaldehyde for $3 \mathrm{hr}$ in phosphate buffer, $\mathrm{pH}$ 7.4 , overnight at $4^{\circ} \mathrm{C}$.

For cryostat sections, cochleae were cryoprotected in $20 \%$ sucrose in PBS, embedded in OCT compound (Tissue Tek; Miles, Elkhart, IN), and mounted for sectioning. For whole-mount surface preparation, cochleae were dissected from all of the surrounding tissue to expose the developing sensory epithelia. RNA probes were synthesized from cDNA for Hes1 (Tomita et al., 1996) and Hes5 (Akazawa et al., 1992). Hes1 was linearized by XhoI and transcribed by T3, and Hes 5 was linearized by HindIII and transcribed by T3 RNA polymerase.

Both whole-mount surface preparations and cryostat sections $(10 \mu \mathrm{m})$ were processed with a digoxigenin (DIG)-labeled cRNA probes as described previously (Schaeren-Wiemers and Gerfin-Moser, 1993). In brief, after fixation, the samples were incubated in 1\% Triton X-100 and digested with Proteinase K. They were incubated in a prehybridization solution for $2 \mathrm{hr}$ at $50^{\circ} \mathrm{C}$ and then exposed to cRNA probes overnight at $50^{\circ} \mathrm{C}$. The probes were revealed by alkaline-phosphatase-coupled antiDIG antibodies (Boehringer Mannheim, Mannheim, Germany), which were reacted with nitroblue-tetrazolium-chloride and 5-bromo-4-chlorindolyl-phosphate substrates for color reaction. Some of the wholemount cochlear preparations were cryosectioned after the in situ hybridization procedures.

Histology and immunocytochemistry. Cochleae from embryos (E16E18) and P0 mice were fixed overnight in $4 \%$ paraformaldehyde in PBS at $4^{\circ} \mathrm{C}$. We performed immunofluorescence analysis on whole-mount surface preparations and paraffin sections of the cochleae as described previously (Zine et al., 2000a). Both cochlea paraffin sections and surface preparations were preincubated in PBS containing 5\% normal donkey serum for $2 \mathrm{hr}$ and then incubated with polyclonal antibodies against myosin V IIa (Hasson et al., 1995) or against Math1 (Helms and Johnson, 1998). Antibody incubations were done overnight at $4^{\circ} \mathrm{C}$, and binding was visualized with rhodamine or Cy3-conjugated donkey anti-rabbit secondary antibodies (Jackson ImmunoResearch, West Grove, PA). Other cochlear surface preparations were stained with rhodamine-conjugated phalloidin $(5 \mu \mathrm{g} / \mathrm{ml})$ for $45 \mathrm{~min}$ to visualize the pattern of hair cell

\section{E12.5 E14 E15 E17 P0}

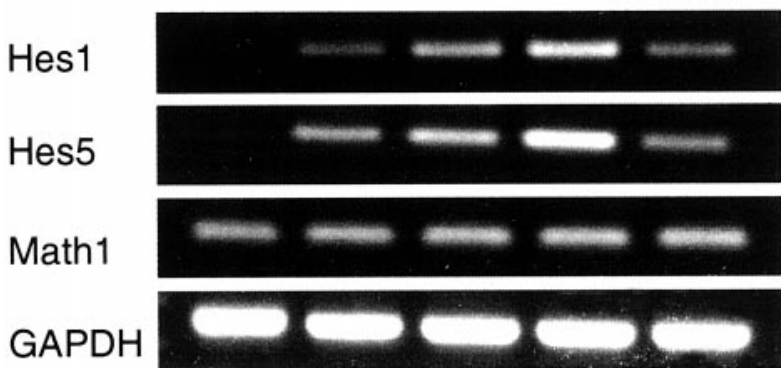

Figure 1. RT-PCR analysis of the temporal expression of Hes1, Hes5, and Math1 in the sensory epithelia dissected from the developing cochleae. All cDNA was coamplified with GAPDH as shown. Math1 transcripts were detected early at E12.5, whereas transcripts for Hes1 and Hes5 were detected by E14. This expression of Hes1, Hes5, and Math1 was maintained in the developing sensory epithelium until P0.

differentiation in the organ of Corti, in particular the actin-rich stereocilia.

Confocal and scanning electron microscopy. Digital images were captured on a Leica (Nussloch, Germany) TCS-NT confocal laser scanning microscope. Images used for the figures were processed with either NIH Image or Photoshop (Adobe Systems, Mountain View, CA) software programs.

For scanning electron microscopy, cochleae were fixed in $2 \%$ glutar-

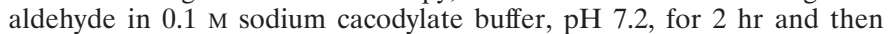
post-fixed for $1 \mathrm{hr}$ with $1 \%$ osmium tetroxide. After washing in cacodylate buffer, cochleae were dehydrated in ascending concentrations of ethanol, dried at the critical point, and sputter coated with gold. All material was examined in a Jeol (Peabody, MA) 630F scanning electron microscope operating at $5 \mathrm{kV}$.

Quantification of hair cells. We quantified the number of cochlear hair cells in early neonatal (P0) mutant mice versus wild-type littermates. Count was made on cochlear surface preparations processed with rhodamine-conjugated phalloidin. We made analysis on a region that covers a $1600 \mu \mathrm{m}$ length of the organ of Corti, beginning near the base of cochlea and extending toward the middle turn.

In the vestibular sensory epithelia, we counted, using the $40 \times$ objective lens, the number of hair cells of P0 utricle and saccule maculae of mutants versus those of wild-type mice on transverse sections immunostained with myosin VIIa across the entire section length of the sensory epithelium. Only segments in which the section was perpendicular to the surface of the neuroepithelium were counted. Hair cell counts were made from three to five distant sections for each macula, and two to five animals were counted for each genotype.

\section{RESULTS}

\section{Hes1, Hes5, and Math1 are expressed in the developing cochlea}

RT-PCR analyses (Fig. 1) were performed with dissected sensory epithelia from the developing cochleae that principally consist of the organ of Corti, a part of the basilar membrane, GER, and LER, as described previously (Zine and de Ribaupierre, 1999). Hes 1 and Hes 5 transcripts became detected by E14, a stage just before the initiation of the cytological differentiation of hair cells at E15 (Lim and Anniko, 1985). Math1 was expressed as early as E12.5, consistent with a study reporting the initiation of Math1/ LacZ expression in the embryonic organ of Corti by E12.5 (Bermingham et al., 1999). The expression of Hes1, Hes5, and Math1 between E14 and P0, a developmental period that is critical for initial hair cell differentiation, suggests the possible functional relationship between these genes.

To determine the cellular expression patterns of Hes 1 and Hes 5 genes, we used nonradioactive in situ hybridization on cryosections and whole-mount surface preparations from E18-P0 co- 
Figure 2. Nonradioactive Hes1 $(A-C)$ and Hes5 $(D-F)$ RNA in situ hybridization in the developing mouse cochlea at E18 and P0. $A, D$, In situ hybridization on transverse sections through the base of E18 cochlea. Hes 1 mRNA $(A)$ is expressed in the LER cells and in a large area of GER, except in the organ of Corti $(O C)$. Hes 5 mRNA $(D)$ is expressed in the LER, pillar cells $(P)$, and Deiter's cells at the base of OHCs (area bracketed by arrows). Hes 5 is also found in the inner phalengeal cells (arrowhead), which are adjacent to the inner hair cell region. Hair cells (large arrow indicates IHC; oblique arrows indicate the three rows of OHCs) are lacking Hes 5 hybridization signal. Note the Hes 5 expression in the marginal cells of the stria vascularis (open arrowhead). Asterisk indicates the spiral vessel, a landmark for the location of the organ of Corti at this stage of maturation. $B, E, I n$ situ hybridization on whole-mount surface preparations from the middle turn of the cochlear duct at P0. Expression of Hes1 $(B)$ spans a large area of the GER, as observed in a cochlea section $(A)$. Expression of Hes5 $(E)$ is observed in a relatively narrow band of cells of the GER. There is a faint but specific Hes5 expression in the cells within the LER (arrows). Note the absence of both Hes 1 and Hes 5 hybridization signal from the hair cells (arrowheads indicate the location of IHC row; bracket indicates the OHC rows). $C, F$, Transverse sections through the whole-mount cochlear surface preparations presented in $B$ and $E$, respectively. $C$, This section confirms Hes1 expression in the GER cells and its lack from the cells of the organ of Corti (bracket). Hybridization label for Hes 1 is also located in the LER (arrow). F, Hes 5 expression is restricted to the nonsensory supporting cells (Deiter's cells; arrows) within the organ of Corti. This section also confirms the specific expression of Hes5 is the LER cells (arrowhead). A weak label for Hes5 is observed in the GER region and in cells located near the basilar membrane. Asterisk indicates the spiral vessel. Scale bars, $20 \mu \mathrm{m}$.

chleae (Fig. 2). In situ hybridization on E18 cochlea sections revealed Hes 1 expression principally in two areas, which include the GER and LER. The organ of Corti, which contains the hair cells and supporting cells, was devoid from Hes 1 signal at this stage of maturation (Fig. $2 A$ ), although a weak hybridization signal for Hes 1 was observed in the supporting cells region in the basal cochlear turn of E15 mice, in addition to the GER and LER (data not shown).

Whole-mount cochlear surface preparations at P0 (Fig. 2B) also showed a widespread expression of Hes 1 in the GER area and its lack from the organ of Corti. A section (Fig. 2C) through the whole-mount surface preparation presented in Fig. $2 B$ confirmed Hes 1 expression in the GER and LER and the absence of Hes 1 hybridization signal from the organ of Corti. Hes5 in situ hybridization (Fig. 2D-F) performed on E18-P0 cochleae indicated both overlapping and distinct expression patterns compared with that of Hes1. In the organ of Corti (Fig. 2D,F), Hes5 is expressed in the pillar cells, Deiter's cells, and the inner phalengeal cells. Like Hes1, the expression of Hes 5 was not detected in the hair cells. Hes 5 was also heavily expressed in the cells of the LER (Fig. 2D), although its expression in the GER was confined to a narrow band of cells (Fig. $2 E$ ). Marginal cells of the stria vascularis showed a specific Hes 5 expression.

To define the roles of Hes 1 and Hes 5 genes in hair cell devel- opment, we analyzed cochlear surface preparations and inner ear sections of different genotypes that resulted from $\mathrm{Hes} 1^{+/-}$; Hes $5^{+/-}$or Hes $1^{+/-}$; Hes $5^{-/-}$intercrosses. We obtained eight possible genotypes (Cau et al., 200) and performed hair cells counts on six of them in the case of cochlea (as summarized in Table 1) and four of them in the case of the vestibule (see Fig. 6).

\section{Deletion of Hes1 gene leads to a significant increase in the number of IHCs}

Whole-mount surface preparation of the cochleae from Hes $1^{-/-}$ mice stained with rhodamine phalloidin revealed many regions (38\% of the relative length of the basilar membrane; Table 1) with a nearly complete row of supernumerary IHCs (Fig. 3D-F) compared with wild-types ( $<1 \%$ of basilar membrane; Table 1$)$, which have only a single row of IHCs (Fig. $3 A-C$ ). In addition, a few regions ( $5 \%$ of basilar membrane with a fourth row of OHCs; Table 1) of Hes $1^{-/-}$mutant cochleae presented four instead of three rows of OHCs (Fig. 3D,F). The presence of the supernumerary hair cells was confirmed by immunostaining cross-sections of cochleae with anti-myosin VIIa, a hair cell-specific marker (Fig. $3 E$ ) and by scanning electron microscopy (Fig. 3F). There was a significant increase in the total number of hair cells in cochleae of Hes $1^{-1-}$ mice compared with wild types. This was principally attributable to a significant increase in the number of 
Table 1. Effects of deletion of Hes1 and Hes5 on auditory hair cell differentiation

Relative length of BM with:

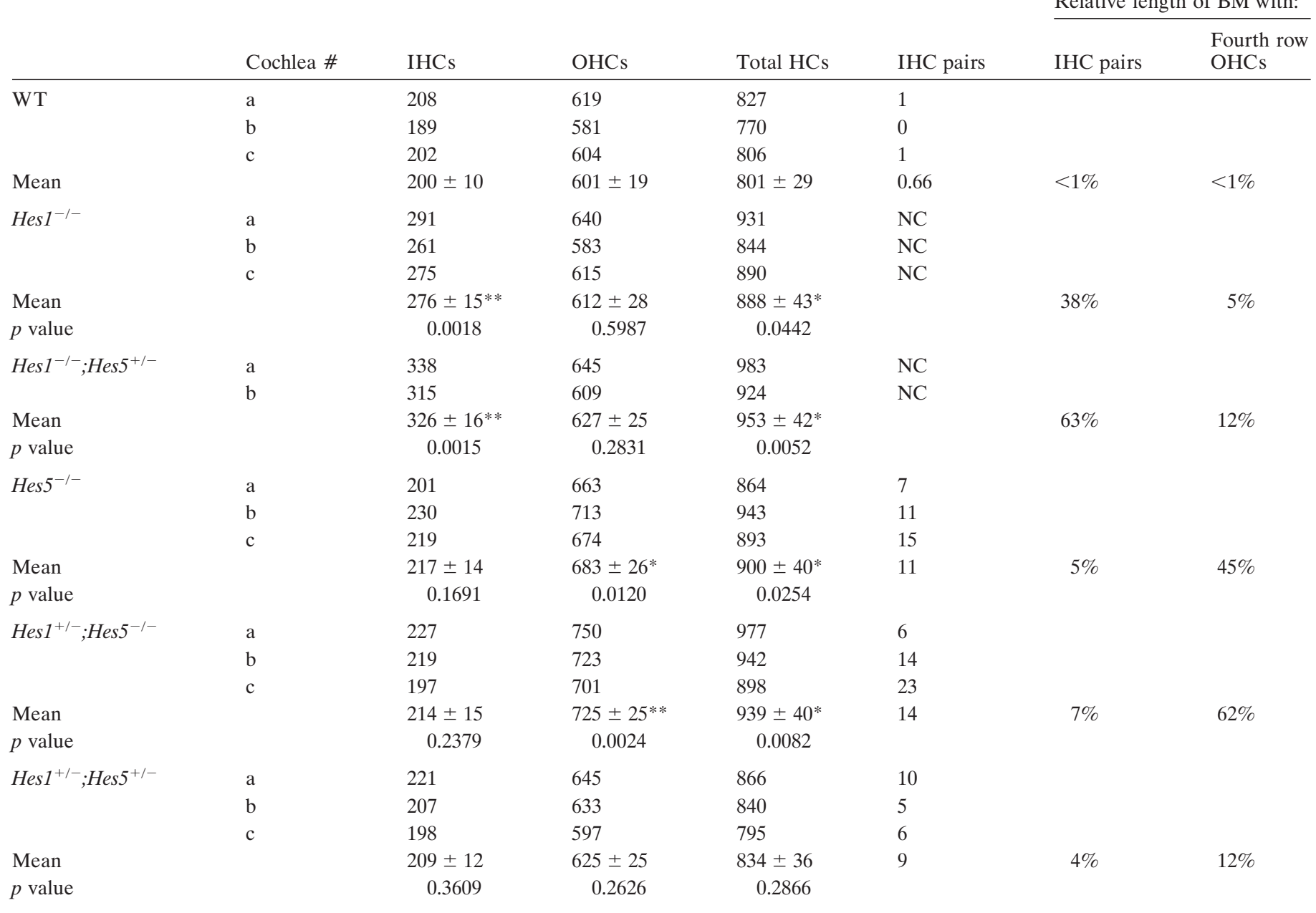

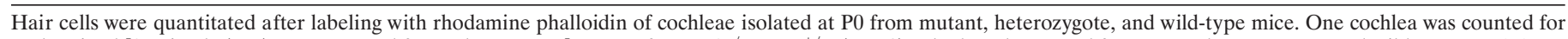

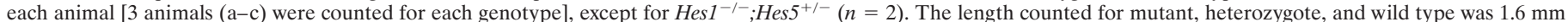

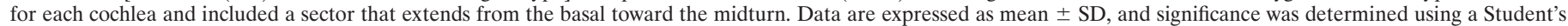

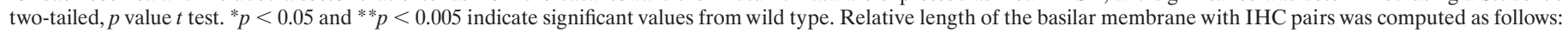

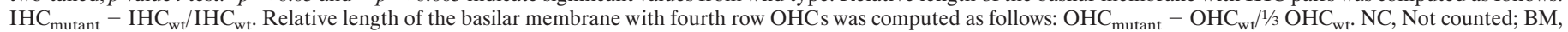
basilar membrane; WT, wild type.

IHCs $(* * p<0.005)$, because the number of OHCs in Hes $1^{-1-}$ cochleae was just slightly higher than wild types (Table 1). The Hes $1^{-1-}$ mice of this study showed a severe cochlea phenotype, with respect to the production of total cochlear hair cells when compared with the results of a recent study (Zheng et al., 2000). This could be attributable to differences in the developmental stage at which hair cell counts were made or to the length of cochlea covered by hair cell counts. In this study, we quantified the number of hair cells in cochleae collected from newborn (P0) mice for either mutant and wild types and along a $1.6 \mathrm{~mm}$ length of the sensory epithelium, i.e., nearly $70 \%$ of the total length.

\section{Deletion of Hes5 gene leads to a significant increase in the number of OHCs}

Cochleae from $\mathrm{Hes} 5^{-1-}$ mice revealed many regions (45\% of the relative length of the basilar membrane with a fourth row of OHCs; Table 1) that principally contained four rows of OHCs rather than three (Fig. 3G-I, Table 1), although, in these mutants, doublets of IHCs ( $5 \%$ of basilar membrane; Table 1$)$ were interspersed within regions of a single row of IHCs (Fig. $3 G, I$ ). Moreover, sometimes cochleae of $\mathrm{Hes} 5^{-1-}$ mice exhibited a few supernumerary hair cells scattered outside the sensory epithelium in the GER (these extra hair cells at this location were not included in hair cells counts), as defined by phalloidin (Fig. 4A) and myosin VIIa (Fig. 4B-D) labeling. In contrast to Hes $1^{-1-}$ mice, the significant increase in the total number of hair cells in the cochleae of $\mathrm{Hes} 5^{-/-}$mice was attributable to the significant increase in the number OHCs $\left({ }^{*} p<0.05\right)$ compared with wild types (Fig. 3G-I, Table 1).

We also examined the cochleae of $H e s 5^{-/-}$mice during the postnatal development because, Hes 5 single mutants were viable. We observed that the supernumerary hair cells survived until P10 (the latest stage we examined) and also developed morphological characteristics of the normal hair cells (data not shown).

\section{Enhanced increase of hair cell production in $\mathrm{Hes1}^{-/-}$; Hes5 $^{+/-}$and Hes1 ${ }^{+/-} ;$Hes5 $5^{-/-}$mutants}

Cochleae from double mutant that were homozygous for Hes 1 and heterozygous for Hes5 $\left(\mathrm{Hes} \mathrm{1}^{-1-} ; \mathrm{Hes} 5^{+/-}\right.$) and those heterozygous for Hes1 and homozygous for Hes 5 (Hes $1^{+/-}$;Hes $5^{-/-}$) mice showed a more significant increase in the total number of 
Figure 3. A comparison of hair cell development in $\mathrm{P} 0$ cochleae from wildtype control $(A-C)$, Hes $1^{-/-}(D-F)$, Hes $5^{-1-}(G-I)$, and Hes $1^{-1-} ;$ Hes $5^{+/-}$ $(J)$ mutants and Hes $1^{+/-} ; \mathrm{Hes}^{+/}$ heterozygous mice $(K)$. Lack of Hes 1 and Hes5 causes the development of supernumerary hair cells. $A, D, G, J$, $K$, Confocal images of surface preparations stained with rhodamine phalloidin to visualize the actin-rich stereocilia of the hair cells. $B, E, H$, Cross-sections through the organ of Corti in the midmodiolar region immunostained with antibody antimyosin VIIa. $C, F, I$, Scanning electron microscopy of the surface of the organ of Corti in the midcochlear turn. In control cochlea, the normal pattern is well defined, with a single row of IHCs and three rows of OHCs. In contrast, in $\mathrm{Hes} 1^{-1-}$ cochleae, two rows of IHCs and three to four rows of $\mathrm{OHCs}$ are present. In Hes 5 cochleae, four rows of $\mathrm{OHCs}$ are often present, in addition to dispersed regions along the sensory epithelium that contain few IHC pairs. $J$, Hes $1^{-1-} ;$ Hes $5^{+/-}$cochleae revealed the same phenotype as Hes $1^{-1-}$, but the effect on the number of supernumerary hair cells was more important in Hes $1^{-1-}$;Hes $5^{+/-}$cochleae (see Table. 1). $K$, Cochlear surface preparation from double heterozygous mouse cochlea indicating regions with extra OHC rows and a few pairs of IHCs. Brackets mark the OHCs rows, and arrowheads point to the IHC row. Scale bars: $A, B, D, E, G, H, J, K, 20 \mu \mathrm{m} ; C, F, I, 10 \mu \mathrm{m}$.

Figure 4. Formation of supernumerary hair cells in both the organ of Corti and the GER in P0 cochlea of Hes5-deficient mice. $A$, Cochlear surface preparation stained with phalloidin, revealing pairs of IHCs in the organ of Corti (arrowheads), in addition to some extra differentiating hair cells with immature stereocilia scattered in the GER (arrows). B, Cross-section through the midmodiolar region of the organ of Corti immunostained with anti-myosin V IIa. Arrows indicate the two differentiating extra hair cells outside the organ of Corti, in the GER area. Note also the extra hair cells in the organ of Corti. $C$, Cochlear surface preparation immunostained with myosin VIIa, indicating the presence of differentiating extra hair cells in the GER (arrows). $D$, Highmagnification of the extra hair cells seen in the GER in $C$ showing their characteristic shape and basal nuclei. Scale bars, $20 \mu \mathrm{m}$.
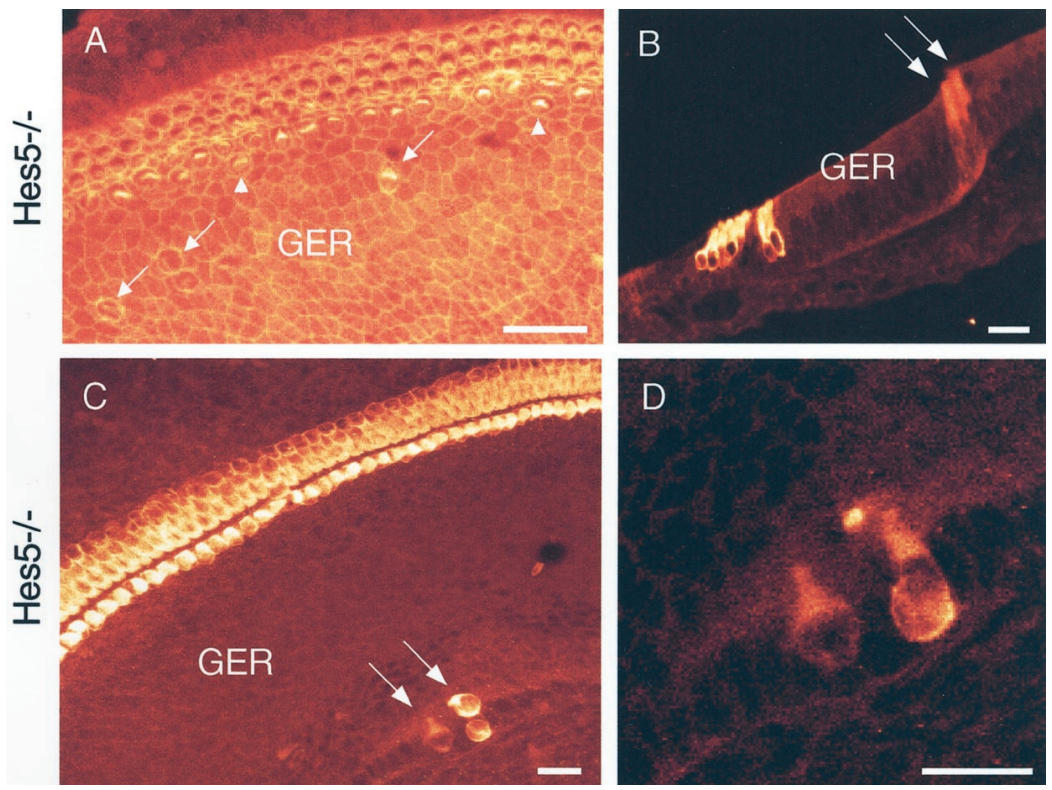

hair cells than Hes 1 and Hes 5 single mutants when compared with the values of wild types (Table 1). This increase in the number of hair cells was attributable to a significant increase in the number of IHCs $\left({ }^{* *} p<0.005\right)$ in Hes $1^{-/-} ;$Hes $5^{+/-}$mice and in the number of OHCs $\left({ }^{* *} p<0.005\right)$ in Hes $1^{+/-} ;$Hes $5^{-/-}$mice compared with wild types (for IHCs: wild type, $200 \pm 10$, mean \pm $\mathrm{SD}$; Hes $1^{-/-}, 276 \pm 15$ Hes $^{-/-}$;Hes $5^{+/-}, 326 \pm 16$ ) (for OHCs: wild type, $601 \pm 19 ;$ Hes $^{-/-}, 683 \pm 26 ;$ Hes $^{+/-} ;$Hes $5^{-/-}, 725 \pm$
25). In addition, the length of the basilar membrane (Table 1) with IHC pairs increased from $38 \%$ in $\mathrm{Hes}^{-1-}$ to $63 \%$ in Hes $1^{-1-}$;Hes $5^{+/-}$mice and that with a fourth row of OHCs increased from $45 \%$ in $\mathrm{Hes} 5^{-/-}$to $62 \%$ in $\mathrm{Hes} \mathrm{1}^{+/-}$; Hes5 $5^{-1-}$. This pronounced defect in $\mathrm{HeS}^{-/-}$;Hes $5^{+/-}$and $\mathrm{Hes} \mathrm{1}^{+/-}$; Hes $5^{-1-}$ mutant cochleae suggests that the dosage of both Hes 1 and Hes 5 gene products is important for the control of hair cell production. There was a mild increase, statistically insignificant, 


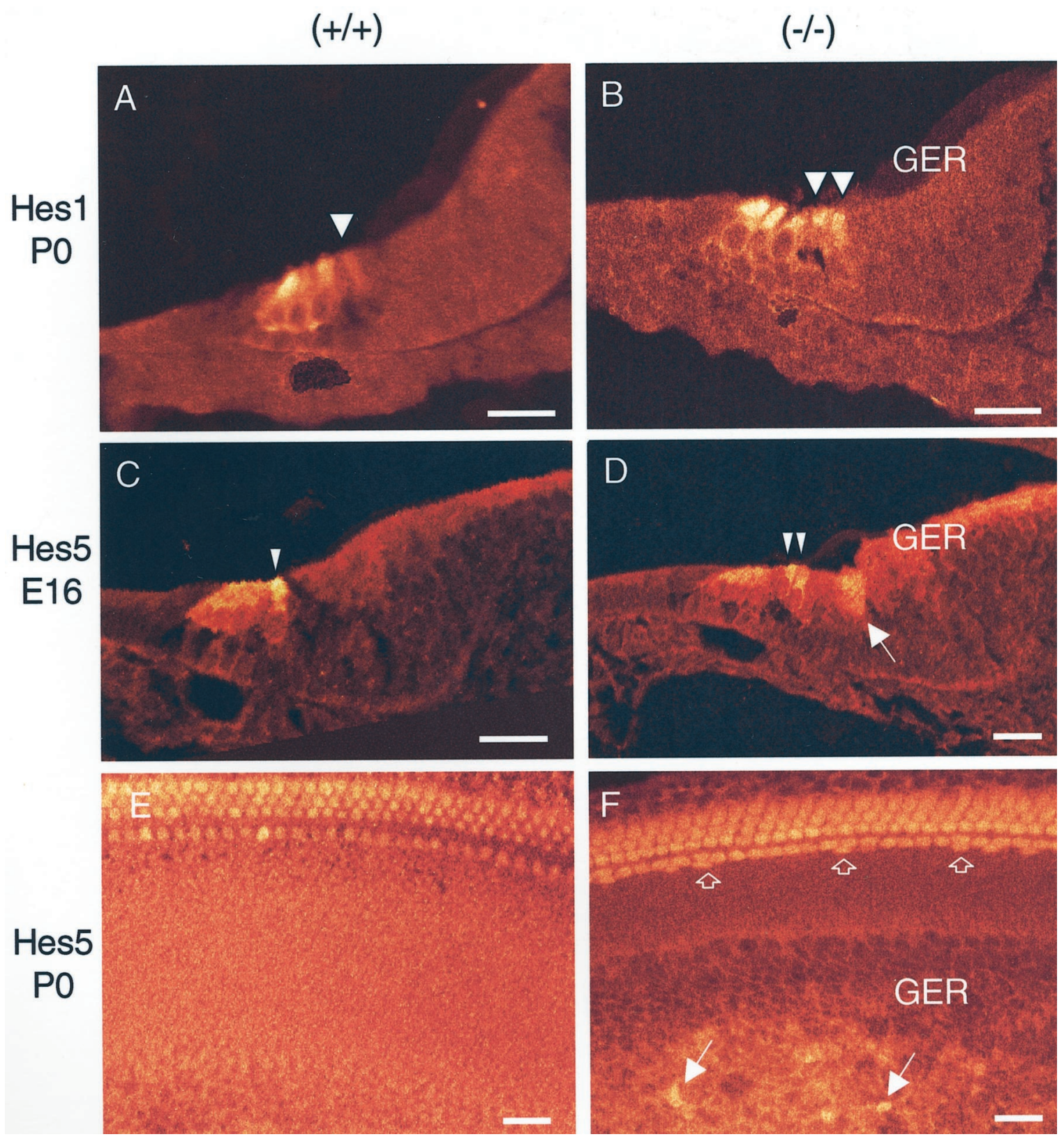

Figure 5. Expression patterns of Math1 protein in the developing cochleae of wild-type (control) $(A, C, E)$, Hes1-deficient (B), and Hes5-deficient $(D$, $F)$ mice. $A, B$, Cross-sections through the midturn of $\mathrm{P} 0$ cochlea from control and Hes $1^{-1-}$ mice. In control, a single inner hair cell (arrowhead) and three outer hair cells express Math1. In Hes $1^{-1-}$, Math1 expression also included the extra IHCs (arrowheads). C, D, Cross-sections of the midturn E16 cochleae from control and Hes $5^{-1-}$ mice showing the differentiating extra hair cells (arrowheads). Note a diff use expression of Math1 in the apical region of some cells in the GER of Hes $5^{-1-}$ mice $($ arrow $) . E, F$, Cochlear surface preparations obtained from P0 control and Hes5 $5^{-1-}$ mice. In control, one row of IHCs and three rows of OHCs express Math1. In Hes $5^{-1-}$, Math1 expression highlights the differentiating extra hair cells in both the organ of Corti (open arrows) and the GER (arrows). Scale bars, $20 \mu \mathrm{m}$.

in the number of hair cells in the double heterozygote $\left(H e s 1^{+/-}\right.$; Hes $5^{+/-}$) mice compared with wild types (Fig. $3 K$, Table1). Because double homozygous embryos for Hes1 and Hes 5 die before E11.5 (Ohtsuka et al., 1999), well before the formation of the cochlea, we were unable to assess hair cell differentiation in double null mutant mice.

\section{Analysis of Math1 expression in the cochleae of Hes1- and Hes5-deficient mice}

Because Math1 has been shown (Bermingham et al., 1999) to be required for the genesis of hair cells, we examined by immunohistochemistry whether the supernumerary hair cells in Hes 1 and
Hes5 mutants mice also expressed Math1 (Fig. 5). Antibody anti-Math1 (Helms and Johnson, 1998) was used to detect Math1 expression in wild-type, Hes1, and Hes 5 mutant cochleae. In wild-types, Math1 expression was restricted to the differentiating hair cells at E16 (Fig. 5C) and by $\mathrm{P} 0$ (Fig. 5A,E) was but absent in the supporting cells and in cells outside the sensory epithelium. Interestingly, in $\mathrm{Hes}^{-/-}$(Fig. 5B) and Hes5 ${ }^{-1-}$ (Fig. 5D) mutant cochleae, Math1 expression also included the supernumerary hair cells that developed within the sensory epithelium. The presence of supernumerary hair cells in the sensory epithelium of E16 Hes $5^{-1-}$ (Fig. 5D) and E16 Hes $1^{-1-}$ mice (data not shown) at the 
earliest developmental time points at which these cells can be distinguished suggests that Hes 1 and Hes 5 genes may have roles in initial steps of hair cell determination-differentiation. In addition, Math1 expression was seen in a subset of extra hair cells that developed outside the organ of Corti, in the GER of Hes5 ${ }^{-1-}$ cochlea (Fig. $5 F$ ). This upregulation of Math1 in differentiating extra hair cells of Hes $1^{-/-}$and Hes $5^{-1-}$ mutant cochleae is consistent with a study demonstrating that Math1 is necessary for the genesis of hair cells in the mice inner ear (Bermingham et al., 1999). Moreover, it was demonstrated in a recent gain-of-function study that overexpression of Math1 in postnatal cochlear cultures resulted in extra hair cells observed in the GER (Zheng and Gao, 2000).

\section{Hes1 and Hes5 genes are also involved in hair cell differentiation in the utricle and saccule sensory epithelia}

To analyze the effects of targeted deletion of Hes 1 and Hes5 on hair cell differentiation within the mouse vestibular organs, we counted the number of cells within the lumenal hair cell layer of P0 utricle and saccule on serial sections, immunolabeled with anti-myosin VIIa (Fig. 6A-D). This quantitation (Fig. 6E,F) showed a significant increase in the mean number of hair cells per unit length of sensory epithelium in the utricle (Fig. $6 F$ ) of Hes $1^{-1-}$ mice $(18.7 \pm 4.4$, mean $\pm \mathrm{SD} ; p<0.0001)$ compared with the wild-type control mice $(12.8 \pm 1.2)$. The number of hair cells in the utricle of Hes $5^{-1-}$ mice $(16.2 \pm 3)$, although lower than that in the utricle of Hes $1^{-/-}$mice, represents a significant increase over the value of wild-type control mice. The number of hair cells in the double heterozygote $\left(\mathrm{Hes} \mathrm{1}^{+/-} ; \mathrm{Hes} 5^{+/-}\right)$utricles was below that of Hes 1 and Hes 5 single mutants and slightly higher than that of the wild-type utricle mice. In the saccule (Fig. $6 E$ ), hair cell differentiation is similarly affected by Hes 1 and Hes 5 deletions as in the utricle. We found a significant, although reduced relative to the value in the utricle, increase in the number of hair cells in Hes $1^{-/-}(16.5 \pm 1.7)$ and Hes5 $5^{-/-}(14.9 \pm 1.9)$ mice when compared with the wild-type $(11.1 \pm 1.3)$ saccule mice.

Our quantitative data from the utricle of $\mathrm{Hes} \mathrm{1}^{-1-}$ mice are in the same range as those reported by Zheng et al. (2000). Their total hair cells counts showed an increase of $36 \%$ in the Hes ${ }^{-1-}$ utricles compared with those from the wild types. This value is very close to the increase in hair cell density that we found for the utricle (38\% increase for Hes $1^{-1-} ; 20 \%$ for Hes $5^{-1-}$ ). In the case of the saccule, our counts indicate an increase of $34 \%$ for $H e s 1^{-/-}$and of $21 \%$ for Hes $5^{-/-}$when compared with the wild types.

\section{DISCUSSION}

Our experiments demonstrate that Hes 1 and Hes 5 have separate and overlapping roles in regulating the differentiation of hair cells in the mammalian inner ear. In the cochlea, Hes 1 has a significant influence on the production of IHCs, whereas Hes5 has a significant influence on the production of OHCs, although a few differentiating extra hair cells were seen in the GER of some cochleae of $\mathrm{Hes} 5^{-/-}$mice. In the vestibular organs, targeted deletion of either Hes 1 or Hes 5 leads to the formation of supernumerary hair cells in both the utricle and saccule sensory epithelia.

Our RT-PCR analysis with isolated cochlear sensory epithelia indicates that Hes1, Hes5, and Math1 genes are expressed during a developmental period that is critical for initial hair cell differ-
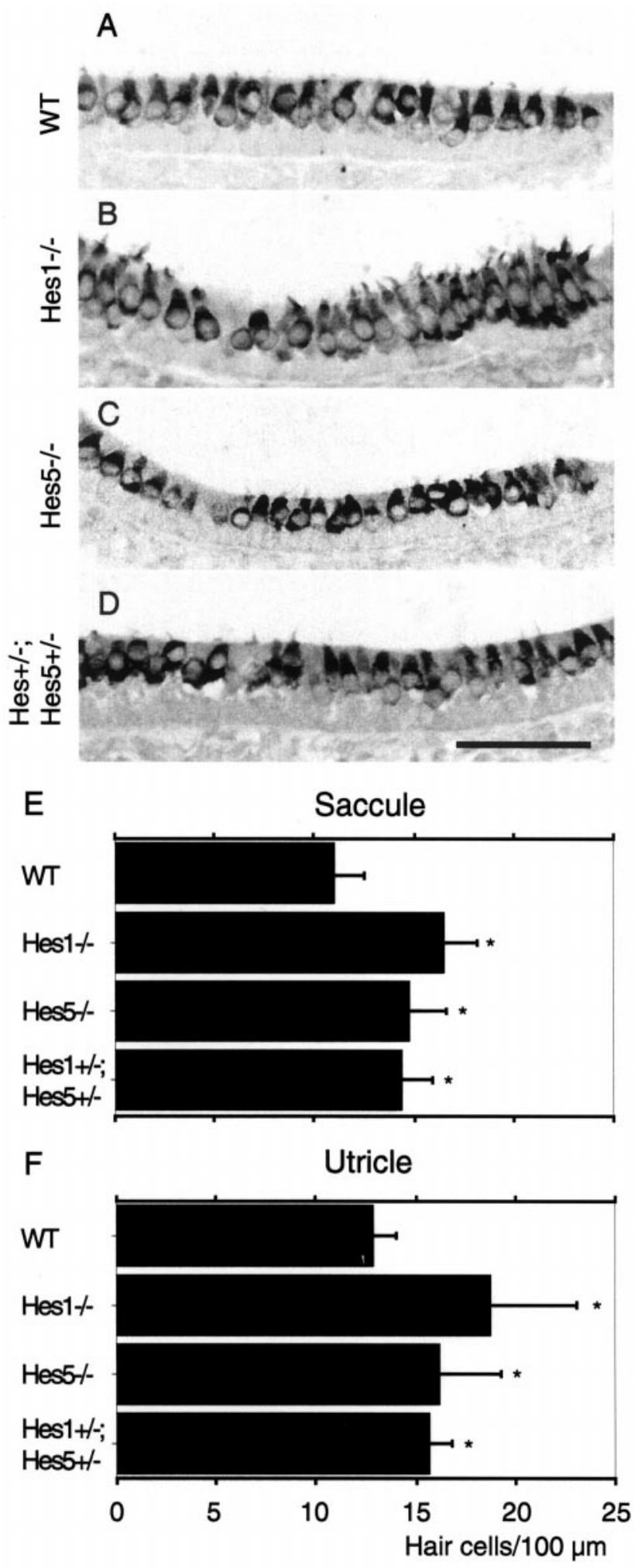

Figure 6. Formation of supernumerary hair cells in the utricle and saccule of Hes1- and Hes5-deficient mice. Myosin VIIa immunolabeling of P0 saccular section from wild-type $(W T)$ control $(A), H_{e s} 1^{-1-}(B)$, Hes $5^{-/-}(C)$, and Hes $1^{+/-} ;$Hes $5^{+/-}$heterozygous mice $(D)$. E, F, Hair cell counts from Hes $1^{-/-}$, Hes $5^{-/-}$and Hes $1^{+/-} ; \mathrm{Hes} 5^{+/-}$mouse saccules and utricles, respectively. Hair cell densities are expressed as a mean number per $100 \mu \mathrm{m}$ length of the sensory epithelium cut perpendicular to the epithelial surface. Data are expressed as mean $\pm \mathrm{SD}$, and significance was determined using a Student's $t$ test. ${ }^{*} p<0.0001$ indicates significant values from wild type. Scale bar: $A-D, 50 \mu \mathrm{m}$. 
entiation. Our results using in situ hybridization with E18-P0 mouse cochleae revealed Hes 1 expression in the GER and LER regions, whereas undetectable to a low level of Hes 1 expression was seen in the supporting cells of the basal turn of E15 cochlea. Hes 5 expression predominated in the LER and in supporting cells of the sensory epithelium of E18-P0 cochleae. In addition, in situ hybridization with whole-mount preparations of the developing cochlea showed a narrow band of Hes5-expressing cells in the GER area (Fig. 2E). These expression data are consistent to some extent with the phenotypic characterization of Hes 1 and Hes 5 mutant cochleae. Indeed, during embryonic development, IHCs derive from progenitor cells located in the most distal domain of the GER, whereas OHCs derive from progenitor cells located in the proximal domain of LER (Lim and Rueda, 1992).

It is interesting that Hes 5 was also expressed in a narrow band of cells within the GER, as in some of the Hes $5^{-1-}$ cochleae in which a few ectopic hair cells were observed in this region, in addition to differentiation of the fourth row of OHCs that arose right next the normal rows. Within the vestibular system, either Hes 1 or Hes 5 deletions induced the formation of supernumerary hair cells in both the utricle and saccule epithelia. This suggests an overlapping role Hes 1 and Hes 5 genes in preventing the formation of supernumerary utricular and saccular hair cells during the normal development of the inner ear. Our quantitative data of the vestibular system indicated that, in Hes 5 mutant mice, the formation of extra hair cells, although significant compared with the wild-type mice, was less important than that of Hes 1 mutant mice. This could fit with the expression study in the utricular epithelium of the rat (Zheng et al., 2000) demonstrating that, unlike Hes 1, which is expressed in the supporting cells throughout the sensory epithelium, Hes 5 expression was confined to the supporting cells in the central area of the utricle (striola). In contrast to the vestibular system, Hes1 expression in E18-P0 mouse cochlea was undetectable in the supporting cells. This suggest that Hes1 may act differently to regulate hair cell differentiation in the inner ear neuroepithelia.

The upregulation of Math1 in the sensory epithelia of Hes1 and Hes5 mutant cochleae (Fig. 5) suggests that Hes genes regulate hair cell differentiation, possibly by antagonizing Math1. This may also occur within the vestibular system because it has been shown that Hes 1 and Hes 5 are expressed in the supporting cell layer of the developing utricle (Zheng et al., 2000). In addition, Math1 is expressed in the vestibular hair cells and is also required for their differentiation (Bermingham et al., 1999; Shailam et al., 1999).

These results are consistent with previous studies demonstrating that the transcription of Math1 is repressed by the transcriptional activities of Hes genes (Takebayashi et al., 1994; Akazawa et al., 1995), although the function of Hes 5 as a repressor of Math1 transcription remains speculative. The increase in cochlear hair cell production in $\mathrm{Hes}^{-/-}$;Hes $5^{+/-}$and Hes ${ }^{+/-}$; Hes $5^{-1-}$ mutant mice compared with Hes 1 and Hes 5 single mutant mice suggests that Hes 1 and Hes 5 genes operate in a common signaling pathway and may functionally compensate for each other. Recent studies have demonstrated that activation of Notch results in the subsequent activation of Hes 1 and Hes 5 genes (Jarriault et al., 1995, 1998; Ohtsuka et al., 1999). Notch proteins are ligands-activated transmembrane receptors involved in cell fate selection throughout development of both Drosophila and vertebrates (Artavanis-Tsakonas et al., 1999).

Studies from several laboratories have reported that the Notch pathway is involved in the development of the vertebrate inner ear (Adam et al., 1998; Haddon et al., 1998; Lanford et al., 1999;
Morrison et al., 1999; Eddison et al., 2000; Zhang et al., 2000; Zine et al., 2000a). In the mammalian cochlea, targeted deletion of Jagged 2 gene, which encodes one of the Notch ligands, resulted in supernumerary hair cells (Lanford et al., 1999). Additionally, alteration of Notch signaling by either Notch1 or Jagged1 antisense oligonucleotides resulted in an increase in the number of hair cells in organotypic cultures of the developing cochlea (Zine et al., 2000a). These similarities in phenotypes when Notch1 and Hes1/Hes 5 functions are altered suggest that Notch1 and Hestype factors act in the same pathway during cochlea development.

In comparison, the expression of Hes 5 and to a lesser extend that of Hes 1 in the developing cochlea is quite similar to that of Notch1 and Jagged1 in terms of their spatial distribution. Previous studies have shown that both Notch1 and its ligand Jagged1 were expressed throughout the prospective cochlear sensory epithelium before hair cell differentiation (Morrison et al., 1999; Zine et al., 2000a). Their expression was downregulated in the differentiating hair cells and persisted in supporting cells and in undifferentiated cells within the GER and LER of the developing cochlea. Altogether, these results may support the role of Hes 1 and Hes5 as downstream mediators of Notch1 signaling pathway in the cochlea and a model in which Notch1-mediated lateral inhibition participates in the regulation of hair cell differentiation. However, phenotypic characterization of both Hes 1 and Hes 5 null mutant cochleae suggests that the function of these Hes genes is not restricted to a lateral inhibitory mechanism between neighboring cells. We did not observe regions of the organ of Corti displaying only hair cells without interposed supporting cells in Hes $1^{-1-}$ and Hes $5^{-/-}$mice, as would be expected if the Hes1 and Hes 5 genes operate only in a lateral inhibitory manner. According to the early expression of Hes 1 in the embryonic mouse cochlea at E14 (Fig. 1), in combination to its broad expression throughout cells of the GER and LER (Fig. 2), Hes1 might also serve as a prepatterning gene, acting to demarcate sensory epithelium versus nonsensory epithelium progenitor cell domains against which determination-differentiation cues are directed. In contrast to Hes1, Hes 5 expression was observed, in addition to GER and LER, in the supporting cells, which includes Deiter's cells and pillar cells within the sensory epithelium of E18 cochlea (Fig. $2 D$ ). This spatial distribution of Hes 5 in the cell types that are closely apposed to the differentiating OHCs may support a role of Hes 5 in Notch-mediated lateral inhibition because Deiter's cells and pillar cells also express Notch1 receptor at this stage of maturation (Zine et al., 2000a).

Such a system parallels to some extent a role of their Drosophila homolog genes, the hairy and $[E(s p l)]$ complex in the generation of mechanosensory bristles that resemble the basic feature of vertebrate mechanosensory hair cells (Chan and Yan, 1999). In Drosophila, the hairy gene participates in proneural cluster positioning as a prepatterning gene and the $[E(s p l)]$ genes act at a later stage in the repression of local neuronal fates within the proneural cluster. This later function is dependent on Notch activation of the genes of the $[E(s p l)]$ complex. In both cases, the effect of hairy and $[E(s p l)]$ complex genes is the negative regulation of neurogenesis through repression of $b H L H$ genes, such as atonal (a Drosophila homolog of Math1) and the achaete-scute complex (Jennings et al., 1994; Fisher and Caudy, 1998). Therefore, Hes 1 and Hes 5 may have Notch-dependent and/or Notchindependent activities in the developing mammalian inner ear, as is possible with their Drosophila homologs, and raise the hypothesis that the function of Notch-Hes-Math1 pathway has been evolutionarily conserved. 
Our findings demonstrate that Hes1 and Hes5 activities are important for repressing the commitment of progenitor cells to IHCs and OHCs fates, respectively, likely by antagonizing Math1. This negative regulation is critical for the correct number of hair cells to be produced and for the establishment of the normal cochlear mosaic of a single row of IHCs and three rows of OHCs. In the vestibular system, Hes 1 and Hes 5 also act as negative regulators of hair cell differentiation within the utricle and saccule epithelia.

A complete understanding of the role of the Notch-HesMath1 pathway directing hair cell fate in the mammalian inner ear may help to develop strategies to solve the problem of auditory hair cells loss. It is possible that simultaneous downregulation of both of Hes1 and Hes 5 in the cochlea might be used to stimulate the replacement of lost auditory hair cells. Such studies may have a significant therapeutic value, because loss of auditory hair cells through disease, trauma, and aging is a common cause of hearing loss and/or deafness.

\section{REFERENCES}

Adam J, Myat A, Le Roux I, Eddison M, Henrique D, Ish-Horowicz D, Lewis J (1998) Cell fate choices and the expression of Notch, Delta and Serrate homologues in the chick inner ear: parallels with Drosophila sense-organ development. Development 125:4645-4654.

Akazawa C, Sasai Y, Nakanishi S, Kageyama R (1992) Molecular characterization of a rat negative regulator with a basic helix-loop-helix structure predominantly expressed in the developing nervous system. J Biol Chem 267:21879-21885.

Akazawa C, Ishibashi M, Shimizu C, Nakanishi S, Kageyama R (1995) A mammalian helix-loop-helix factor structurally related to the products of Drosophila proneural gene atonal is a positive transcriptional regulator expressed in the developing nervous system. J Biol Chem 270:8730-8738

Anniko M (1983) Cytodifferentiation of cochlear hair cells. Am J Otolaryngol 4:375-388.

Artavanis-Tsakonas S, Rand MD, Lake RJ (1999) Notch signaling: cell fate control and signal integration in development. Science 284:770-776

Bermingham NA, Hassan BA, Price SD, Vollrath MA, Ben-Arie N, Eatock RA, Bellen HJ, Lysakowski A, Zoghbi HY (1999) Math1: an essential gene for the generation of inner ear hair cells. Science 284:1837-1841.

Cau E, Gradwhol G, Casarosa S, Kageyama R, Guillemot F (2000) Hes genes regulate stages of neurogenesis in the olfactory epithelium. Development 127:2323-2332.

Chan Y-M, Yan YN (1999) Conservation of neurogenic genes and mechanisms. Curr Opin Neurobiol 9:582-588.

Eddison M, Le Roux I, Lewis J (2000) Notch signaling in the development of the inner ear: lessons from Drosophila. Proc Natl Acad Sci USA 97:11692-11699.

Fekete DM (1996) Cell fate specification in the inner ear. Curr Opin Neurobiol 6:533-541.

Fisher A, Caudy M (1998) The function of hairy-related bHLH repressor proteins in cell fate decisions. BioEssays 20:298-306.

Haddon C, Jiang YL, Smithers L, Lewis J (1998) Delta-Notch signalling and the patterning of sensory cell differentiation in the zebrafish ear: evidence from the mind bomb mutant. Development 125:4637-4644.

Hasson T, Heintzelman MB, Santos-Sacchi J, Corey DP, Mooseker MS (1995) Expression in cochlea and retina of myosin VIIa, the gene product defective in Usher syndrome type 1B. Proc Natl Acad Sci USA 92:9815-9819.

Helms AW, Johnson JE (1998) Progenitors of dorsal commissural interneurons are defined by MATH1 expression. Development 125:919-928.

Ishibashi M, Ang SL, Shiota K, Nakanishi S, Kageyama R, Guillemot F (1995) Targeted disruption of mammalian hairy and Enhancer of split homolog-1 (Hes-1) leads to upregulation of neural helix-loop-helix factors, premature neurogenesis, and severe neural tube defects. Genes Dev 9:3136-3148.

Jarriault S, Brou C, Logeat F, Schroeter E, Kopan R, Israel A (1995)
Signalling downstream of activated mammalian Notch. Nature 377:355-358.

Jarriault S, Le Bail O, Hirsinger E, Pourquie O, Logeat F, Strong C, Brou C, Seidah N, Israel A (1998) Delta-1 activation of Notch-1 signaling results in Hes-1 transactivation. Mol Cell Biol 18:7423-7431.

Jennings B, Preiss A, Delidakis C, Bray S (1994) The Notch signaling pathway is required for Enhancer of Split bHLH protein expression during neurogenesis in the Drosophila embryo. Development 120:3537-3548.

Kageyama R, Nakanishi S (1997) Helix-loop-helix factors in growth and cell differentiation of the vertebrate nervous system. Curr Opin Genet Dev 7:659-665.

Kelley MW, Xu XM, Wagner MA, Warchol ME, Corwin JT (1993) The developing organ of Corti contains retinoic acid and forms supernumerary hair cells in response to exogenous retinoic acid in culture. Development 119:1041-1053.

Lanford PJ, Lan Y, Jiang R, Lindsell C, Weinmaster G, Gridley T, Kelley MW (1999) Notch signaling pathway mediates hair cell development in mammalian cochlea. Nat Genet 21:289-292.

Lee JE (1997) Basic helix-loop-helix genes in neural development. Curr Opin Neurobiol 7:13-20.

Lim DJ, Anniko M (1985) Developmental morphology of the mouse inner ear. A scanning electron microscopy observation. Acta Otolaryngol 422:1-69.

Lim D, Rueda J (1992) Structural development of the cochlea. In: Development of auditory and vestibular systems 2 (Romand R, ed), pp 33-58. New York: Elsevier.

Morrison A, Hodgetts C, Gossler A, Hrabé de Angelis M, Lewis J (1999) Expression of Delta1 and Serrate1 (Jagged1) in the mouse inner ear. Mech Dev 84:169-172.

Ohtsuka T, Ishibashi M, Gradwohl G, Nakanishi S, Guillemot F, Kageyama R (1999) Hes 1 and Hes 5 as Notch effectors in mammalian neuronal differentiation. EMBO J 18:2196-2207.

Ruben RJ (1967) Development of the inner ear of the mouse: a radioautographic study of terminal mitosis. Acta Otolaryngol 220:1-44.

Sasai Y, Kageyama R, Tagawa Y, Shigemoto R, Nakanishi S (1992) Two mammalian helix-loop-helix factors structurally related to Drosophila hairy and Enhancer of split. Genes Dev 6:2620-2634.

Schaeren-Wiemers N, Gerfin-Moser A (1993) A single protocol to detect transcripts of various types and expression levels in neural tissue and cultured cells: in situ hybridization using digoxigenin-labelled cRNA probes. Histochemistry 100:431-440.

Shailam R, Lanford PJ, Dolinsky CM, Norton C Gridley T, Kelley MW (1999) Expression of proneural and neurogenic genes in the embryonic mammalian vestibular system. J Neurocytol 28:809-819.

Takebayashi K, Sasai Y, Sasai K, Watanabe T, Nakanishi S, Kageyama R (1994) Structure, chromosomal locus, and promoter analysis of the gene encoding the mouse helix-loop-helix factor HES1: negative autoregulation through the multiple $\mathrm{N}$ box elements. J Biol Chem 269:5150-5156.

Tomita K, Ishibashi M, Nakahara K, Ang SL, Guillemot F, Kageyama R (1996) Mammalian hairy and Enhancer of split homolog 1 regulates differentiation of retinal neurons and is essential for eye morphogenesis. Neuron 16:723-734

Torres M, Giraldez F (1998) The development of the vertebrate inner ear. Mech Dev 71:5-21.

Van de Water TR (1983) Embryogenesis of the inner ear: in vitro studies. In: Development of auditory and vestibular systems (Romand $\mathrm{R}$, ed), pp 337-374. New York: Elsevier.

Zhang N, Martin GV, Kelley MW, Gridley T (2000) A mutation in the Lunatic fringe suppresses the effects of a Jagged 2 mutation on inner hair cell development in the cochlea. Curr Biol 10:659-662.

Zheng JL, Gao WQ (2000) Overexpression of Math1 induces robust production of extra hair cells in postnatal rat inner ears. Nat Neuroci 3:580-586

Zheng JL, Shou J, Guillemot F, Kageyama F, Gao WQ (2000) Hes1 is a negative regulator of inner ear hair cell differentiation. Development 127:4551-4560.

Zine A, de Ribaupierre F (1999) Tissue specific levels and cellular distribution of epidermal growth factor receptors within control and neomycin-damaged neonatal rat organ of Corti. J Neurobiol 38:313-322.

Zine A, Van de Water TR, de Ribaupierre F (2000a) Notch signaling regulates the pattern of auditory hair cell differentiation in mammals. Development 127:3373-3383.

Zine A, Nyffeler M, de Ribaupierre F (2000b) Spatial expression patterns of epidermal growth factor receptor gene transcripts in the postnatal mammalian cochlea. Hear Res 141:19-27. 